\title{
RELACIÓN ENTRE EL PESO FRESCO Y EL PESO SECO DEL RASTROJO DE MAÍZ EN DIFERENTES ESTADOS FENOLÓGICOS DEL CULTIVO ${ }^{1}$
}

\author{
M. Bänziger ${ }^{2}$, G.O. Edmeades ${ }^{2}$, J. Bolaños ${ }^{3}$
}

\begin{abstract}
RESUMEN
Relación entre el peso freco y el peso seco del rastrojo de maíz en diferentes estados fenológicos del cultivo. En este estudio, se calcularon la relación que existe entre el peso fresco del rastrojo de maíz y el peso seco en varias etapas del crecimiento, con muestras periódicas que se tomaron en dos localidades de ocho cultivares con diferente vigor y periodos de madurez. Las diferencias de los cultivares en el porcentaje de materia seca en el rastrojo (MS\%) fueron más notorias en la segunda mitad del llenado, cuando los cultivares de madurez tardía generalmente contienen menos humedad que los cultivares de madurez precoz. Se hizo la regresión del MS\% contra el estadio de desarrollo fenológico expresado en forma relativa contra la antesis ( $\mathrm{R}$; días transcurridos entre la siembra y el muestreo/días transcurridos desde la siembra hasta el 50\% de la antesis). Las ecuaciones con mejores resultados $(\mathrm{R} 2=0,97-$ 0,99) fueron: Cultivares de maduración precoz: $\mathrm{MS} \%=12,6+$ 0,94R2 + 1,68R4; Cultivares de maduración tardía: MS\% = 16,1 - 4,00R2 + 3,36R4. No hubo diferencias constantes entre los cultivares con diferentes niveles de vigor aunque las diferencias atribuidas a la humedad relativa fueron observadas entre localidades. Se describe un protocolo para determinar el peso seco del rastrojo de maíz por unidad de área ( ha-1) en las fincas de los agricultores, utilizando únicamente una báscula y una regla. Además, se sugiere un método para calcular el porcentaje de materia seca de la parte de la planta por encima del suelo (incluyendo el grano) (MST\%).
\end{abstract}

\begin{abstract}
Relationship between fresh and dry weigth of corn stover at different phenological stages. The amount of dry matter produced during various stages of corn growth is a important variable to be taken into consideration. However, the lack of drying facilities makes its measurement a difficult task in the fields. A simple method to convert the fresh weight of a crop in the field into dry weight, could be an answer to that problem. In this study, we calculated the relationship between fresh and dry weight of corn stovers, over several, growth, stages of eight corn cultivars of different vigour and maturity period, at two Mexican locations. The differences between cultivars were for percent stover dry weight (\%SDW) most evident in the second half of the grain growth stage, when late cultivars showed less humidity than the early ones. The $\%$ SDW was regressed against the phenological developmental stage and expressed as a ratio against antesis ( $\mathrm{R}$, days to sampling /days to $50 \%$ antesis). The equations ( $\mathrm{R} 2=0.97-$ $0.99)$ with best results were: Early maturing cultivars: \%SDW $=12.6+0.94 \mathrm{R} 2+1.68 \mathrm{R} 4$; Late: $\% \mathrm{SDW}=16.1-4.00 \mathrm{R} 2+$ 3.36R4. There were no consistant differences among cultivars with different vigour levels, even though certain differences were noted among the locations and they were attributed to differences in relative humidity. We describe a protocol for determining the dry weight of corn stover by area unit (t/ha) when drying conditions are not available, by utilizing only a scale and a ruler.We also suggest a method to calculate percent dry matter for a real plant parts (including grain).
\end{abstract}

\section{INTRODUCCION}

Es muy útil poder convertir los pesos frescos a pesos secos en finca, utilizando la relación entre el contenido de humedad de la planta y su etapa de crecimiento. Sin embargo, la ausencia de secadoras en los campos de los agricultores dificulta la obtención de este tipo de dato. Las secadoras y hornos existentes se encuentran generalmente en las estaciones experimentales, algunas veces lejos del lugar donde se llevan a cabo los experimentos.

\footnotetext{
1 Presentado en la XLI Reunión Anual del PCCMCA en El Salvador, Centroamérica. 27 al 31 de marzo, 1996.

2 Fisiólogos de Cultivos, CIMMYT, México.

3 Agrónomo/Fisiólogo, Programa Regional de Maíz, CIMMYT, Guatemala
} 
Incluso cuando las instalaciones de secado se encuentran cerca, los problemas para transportar grandes cantidades de material fresco al laboratorio, la necesidad de obtener sub-muestras apropiadas y la capacidad inadecuada de las secadoras, a menudo ocasionan que el investigador no obtenga la información necesaria.

En un cultivo anual y determinado como el maíz (Zea mays L.), la proporción de materia seca cambia a medida que el cultivo atraviesa sus distintos estadios fenológicos de crecimiento. Un método conveniente para comparar, durante la misma etapa de crecimiento, distintos genotipos de maíz con diferentes periodos de madurez, es dividir el tiempo que transcurre entre la siembra y el muestreo entre el tiempo que transcurre entre la siembra y la antesis. Utilizando esta medida, llamada etapa de crecimiento relativo $(\mathrm{R})$, el cultivo alcanza la $\mathrm{R}=0,4$ al inicio del espigamiento, $R=1,0$ en la antesis, $R=1,5$ a la mitad del llenado y $\mathrm{R}=1,9$ cuando se alcanza la madurez fisiológica. La cosecha del grano en la mayoría de los cultivares de maíz se lleva a cabo aproximadamente $\mathrm{R}=2,2$, cuando el contenido de humedad en el grano es de alrededor del $20 \%$.

La proporción de agua en las plantas es alta cuando éstas son jóvenes y disminuye conforme envejecen, alcanzando un nivel mínimo en la madurez. La semilla alcanza un nivel aún inferior de contenido de agua, para así continuar el ciclo. En las plantas jóvenes, el tejido foliar, que tiene un gran contenido de agua debido a sus importantes funciones de intercambio de gases, fotosíntesis y transporte de nutrimientos y minerales, constituye la mayor parte del peso fresco. Si el contenido de agua en las hojas disminuye por debajo de $30 \%$ de su valor máximo debido a la sequía, las hojas morirán (Ludlow y Muchow, 1990; Loomis y Connor, 1992). Conforme la planta crece, el tallo representa una mayor proporción del peso seco y la planta se vuelve más fibrosa ya que aparecen los materiales estructurales como las ligninas y las hemicelulosas con un contenido de humedad más bajo. Aproximadamente de 10 a 14 días después de la polinización, el almidón empieza a acumularse y de manera simultánea, el agua es desplazada del grano. Esto provoca un rápido aumento en el porcentaje de materia seca de la mazorca, del $17 \%$ durante la antesis al $70 \%$ a los 50 días de la polinización aproximadamente $(\mathrm{R}=19)$. Como el peso seco de la mazorca aumenta menos del $1 \%$ del peso seco total de la planta aproximadamente el $50 \%$ durante el mismo tiempo, este incremento en el porcentaje de materia seca se refleja en el contenido de materia seca de toda la planta. Al mismo tiempo, las hojas se volverán más anchas y aumentará el nivel de fibra en los tallos. Juntos, estos cambios dan como resultado un fuerte incremento en el contenido de materia seca de toda la planta durante el llenado de alrededor del $20 \%$ durante la antesis a alrededor del $50 \%$ cuando se alcanza la madurez fisiológica $(\mathrm{R}=1.9)$. En esta etapa, el contenido de materia seca es de aproximadamente el $67 \%$, y el del resto de la planta de alrededor de $42 \%$. Para cuando el contenido de materia seca alcance $80 \%(\mathrm{R}=2,2)$, el de la planta entera será del 65 a 70\% (Daynard y Hunter, 1975; Fairley, 1980). Subsecuentemente, la planta muere y sus componentes, con la excepción del grano, pierden agua rápidamente hasta alcanzar un contenido de materia seca estable de un tres a un ocho porciento menor que el grano unas semanas después de la madurez fisiológica, cuando $R=2,5$. En cualquier etapa del crecimiento, el ambiente puede alterar el porcentaje de materia seca en el cultivo. Si la planta está sometida a una fuerte demanda evaporativa, su contenido de agua puede disminuir un $10 \%$ durante el llenado y tal vez un $15 \%$ en la etapa posterior a la floración (Loomis y Connor, 1992). La muerte prematura del cultivo provocada por la sequía o las heladas también cambia la relación entre la materia seca de la planta y la etapa de crecimiento.

El objetivo del presente trabajo fueron determinar la relación entre el peso fresco y el peso seco del rastrojo por encima del suelo en varias etapas de crecimiento en cultivares con diferentes periodos de madurez cuando se les cultiva en dos localidades diferentes en la temporada de lluvias de verano en México. Durante la mayor parte del ciclo de cultivo, el peso del rastrojo correspondió al peso total por encima del suelo, ya que el peso de la mazorca no fue tomado en cuenta. El estudio fue una actividad conjunta entre el CIMMYT, México y el Programa Regional de Maíz (PRM) de Centroamérica y el Caribe.

\section{MATERIALES Y METODOS}

Los experimentos se llevaron a cabo en las estaciones experimentales de Tlaltizapán (TL) y Poza Rica (PR) en la estación de lluvias de verano (junio-octubre) de 1994. TL se localiza a $19^{\circ} \mathrm{N}$, a una altitud de $940 \mathrm{~m}$. Su temporada de cultivo del verano se caracteriza por valores de temperatura máxima y mínima diarios de $31^{\circ} \mathrm{C}$ y 17 (C y una radiación de $21 \mathrm{MJ} \mathrm{m}^{-2}$. Por el contrario, PR se localiza cerca del Golfo de México a $21^{\circ} \mathrm{N}$ y a $60 \mathrm{~m}$ de altitud. Su temporada de verano tiene valores promedios de temperatura máxima, mínima y de radiación de $32{ }^{\circ} \mathrm{C}$, $22{ }^{0} \mathrm{C}$ y $22 \mathrm{MJ} \mathrm{m}^{-2}$. En general, PR es más húmedo que $\mathrm{TL}$, incluso durante la temporada de lluvias. El suelo en PR es franco arenoso (Troplofluvent) y el de TL es arcilloso calcáreo (Isothermic Udic Pellustert). 


\section{Materiales vegetales}

Se sembraron ocho cultivares: cuatro variedades de polinización libre (VPLs) y cuatro híbridos (Cuadro 1), en un diseño de látice $2 * 4(0,1)$ con tres repeticiones en ambas localidades. Los cultivares representaban diferentes vigores y periodos de madurez típicos de ambientes tropicales en Centroamérica (VPLs contra híbridos). Las parcelas eran de cinco surcos con una separación de 0.75 $\mathrm{m}$ y una longitud de $10,5 \mathrm{~m}$ con una densidad de 5.3 plantas $\mathrm{m}^{-2}$. Al cultivo se le mantuvo libre de maleza, insectos y enfermedades.

Medidas: En siete ocasiones se hicieron recolectas de nueve plantas $\left(1,7 \mathrm{~m}^{2}\right)$ de los tres surcos centrales de cada parcela (de aproximadamente 26 a 40 días después de la siembra; un día después del $50 \%$ de la antesis: 70, 85 y 90 días después de la siembra y en la madurez fisiológica). En cada recolecta para determinar el peso fresco, las plantas fueron cortadas a nivel del suelo aproximadamente a las 10 de la mañana y se les llevó a un laboratorio de campo. El tiempo que transcurrió entre cortar la planta y pesarla fue de menos de 10 minutos. Si las hojas estaban mojadas por la lluvia, la recolecta se posponía hasta que las hojas se hubieran secado. Las plantas de cada parcela se cortaron en pedazos pequeños y se secaron a $80^{\circ} \mathrm{C}$ en una estufa durante cinco días y después se pesaron. Para las primeras cuatro recolectas no se separó ninguna parte de las plantas por lo que el peso del rastrojo de estas recolectas fue el equivalente al peso de la planta por encima del suelo. En las recolectas realizadas 85 y 99 días después de la antesis, se retiraron las mazorcas y solamente se pesó el rastrojo fresco (tallos + hojas + espigas + bracteas), mientras que las mazorcas fueron retiradas, secadas y desgranadas, además de calcularse el rendimiento de grano. Se recolectaron 18 plantas $\left(3,4 \mathrm{~m}^{2}\right)$ en etapa de madurez fisiológica para obtener grano y biomasa. Igualmente, se tomó el peso fresco total del rastrojo y las mazorcas. Cada una de las partes se secó por separado y se calculó el rendimiento del grano.

Se registraron los días que transcurrieron desde la siembra hasta el $50 \%$ de la antesis de 38 plantas útiles en cada parcela. Las fechas de recolecta para calcular la biomasa se expresaron en unidades R (i.e., [días transcurridos desde la siembra hasta la recolecta)/(días transcurridos desde la siembra hasta el 50\% de la antesis]), para confirmar que las comparaciones se hicieran entre cultivares y localidades con una etapa de desarrollo de planta similar. El contenido de materia seca del grano en la madurez también se determinó pesando y secando una muestra de grano de cinco mazorcas localizadas en los surcos de la orilla de cada parcela cuando se llevó a cabo la última recolecta. El rendimiento de grano se determinó por medio de una muestra de 24 plantas por parcela.

\section{Análisis de los datos}

El porcentaje de materia seca del rastrojo (MS\%) se expresó como [100*peso seco/peso fresco] y se llevó a cabo análisis de regresión entre $\mathrm{MS} \%$ contra $\mathrm{R}$ para cada parcela, resultando MS\% $=\mathrm{a}+\mathrm{b}(\mathrm{R}) 2+\mathrm{c}(\mathrm{R}) 4(\mathrm{R} 2=0,97 \mathrm{a}$ $0,99)$ la mejor ecuación. Los coeficientes de regresión a, b, y c de cada parcela en TL y PR fueron sometidos a un análisis combinado de varianza utilizando un modelo fijo. Del mismo modo, se utilizaron valores promedio de los coeficientes para comparar la respuesta de MS\% a R para los cultivares y lugares.

Cuadro 1. Características de ocho cultivares de maíz (cuatro variedades de polinización libre y cuatro híbridos) durante 1994 en Tlaltizapán y Poza Rica, México. El rendimiento de grano es a cero humedad (secado en horno a $\left.80^{\circ} \mathrm{C}\right)$.

\begin{tabular}{lllclll}
\hline $\begin{array}{l}\text { Cultivar } \\
\text { antesis }\end{array}$ & $\begin{array}{c}\text { Vigor } \\
\text { grano }\end{array}$ & $\begin{array}{c}\text { Ciclo } \\
\text { t/ha }\end{array}$ & $\begin{array}{c}\text { Días a } \\
\text { grano }\end{array}$ & Tipo de & Rend gr & \%MS \\
\hline 1. Selección Precoz C6 & VPL & precoz & 50 & $\mathrm{AmCr}$ & 4,46 & 85,1 \\
2. Selección Precoz C16 & VPL & ex-precoz & 47 & $\mathrm{AmCr}$ & 3,93 & 86,1 \\
3. La Posta Sequía C3 & VPL & tardío & 58 & $\mathrm{BlDn}$ & 5,11 & 79,8 \\
4. TS6 C1 (PITS) F2 & VPL & tardío & 59 & $\mathrm{BIDn}$ & 5,31 & 79,7 \\
5. CML20 x CML27 & Hibr CS & tardío & 60 & $\mathrm{AmCr}$ & 5,68 & 78,5 \\
6. CML27 x CML52 & Hibr CS & tardío & 60 & $\mathrm{AmCr}$ & 5,83 & 78,0 \\
7. CML8 x CML9 & Hibr CS & tardío & 61 & $\mathrm{BIDn}$ & 5,12 & 73,4 \\
8. CML8 x CML22 & Hibr CS & tardío & 58 & BIDn & 6,21 & 74,0 \\
\hline
\end{tabular}

$\mathrm{AmCr}=$ amarillo cristalino; B1Dn = blanco dentado 


\section{RESULTADOS Y DISCUSION}

\section{Diferencias de los cultivares}

Una unidad de $\mathrm{R}$ para todo el ensayo fue, en promedio de 56,6 días. Los rendimientos del grano obtenidos (expresados a cero humedad) fueron normales para estas localidades (Cuadro 1). Los contenidos de materia seca del rastrojo por cada cultivar se resumen en el Cuadro 2 y reflejan las diferencias (de hasta 14 días) en la fecha al $50 \%$ antesis de las distintas entradas. Los coeficientes de regresión que se enumeran son los promedios de seis parcelas (2 localidades $\mathrm{X} 3$ repeticiones) y son ligeramente diferentes a los que se adaptaron a la información recopilada de cada cultivar. Las regresiones se usaron para predecir los valores de MS\% para distintas etapas de $\mathrm{R}$ (Cuadro 2). No se detectaron diferencias considerables en los cultivares en el MS\% esperado hasta etapas tardías en el desarrollo del cultivo, cuando R $>1,67$ (corresponde al $70 \%$ del llenado). Las diferencias entre los cultivares a $\mathrm{R}=2,0$ (una semana después de la madurez fisiológica) fueron muy notorias, variando del 40,7\% (Selección Precoz C16) al 60,5\% (La Posta Sequía C3), aunque no hubo diferencias notorias entre los híbridos y las VPLs en el DM\% esperado. Hubo una tendencia de los cultivares de madurez precoz (Selección Precoz C6 y C16) a contener más agua que los cultivares de madurez tardía de $\mathrm{R}=1,67$ hacia arriba. Este hecho podría provocar diferencias considerables entre los grupos de madurez distinta. Esto podría deberse a que los cultivares de madurez tardía estaban madurando cuando las lluvias estaban a punto de terminar y la humedad relativa del aire iba en descenso, mientras que los dos cultivares precoces completaron el llenado cuando todavía había lluvias abundantes. Las VPLs tardías también mostraron una tendencia a tener mayor MS\% hacia el final del llenado que los híbridos, tal vez reflejando su periodo de llenado más corto (Bolaños, 1995) y senescencia más rápida.

\section{Diferencias en las localidades}

El análisis de varianza de los coeficientes de regresión adaptados a parcelas individuales y los valores esperados de MS\% (Cuadro 2) mostraron que TL difería de PR en que las plantas tenían menor MS\% desde la antesis hasta la mitad del llenado y mayor MS\% en cualquier otro periodo fuera de este intervalo. Es probable que esto refleje las diferencias del tiempo que se tomó entre cortar las plantas y calcular los pesos frescos. Es evidente que el intervalo entre el corte de las plantas y el pesarlas debe ser corto pero razonable, ya que las plantas pierden agua a través de las hojas a un paso acelerado, especialmente cuando se encuentran expuestas al sol. La importante interacción cultivar X localidad
Cuadro 2. Coeficientes de regresión de la relación entre el porcentaje de materia seca $(\% \mathrm{MS})$ de la biomasa por encima del suelo y del estadio relativo de desarrollo fenológico $(\mathrm{R})$, definido este como $\mathrm{R}=$ dds al muestro/ dds a antesis, para ocho cultivares de maíz a través de dos localidades en México, 1995. La función usada es del tipo \% MS=a+vr2+CR4.

\begin{tabular}{lrrr}
\hline & a & b & c \\
\hline 1. Sel. Precoz C6 & 13,5 & $-0,65$ & 2,17 \\
2. Sel. Precoz C16 & 11,7 & 2,53 & 1,18 \\
3. La Posta C3 & 16,2 & $-5,01$ & 4,02 \\
4. TS6 C1 (PITS) & 16,8 & $-6,34$ & 4,19 \\
5. CML20 x CML27 & 15,6 & $-5,11$ & 3,91 \\
6. CML27 x CML52 & 16,3 & $-3,05$ & 2,92 \\
7. CML8 x CML9 & 15,7 & $-2,87$ & 2,75 \\
8. CML8 x CML22 & 15,9 & $-1,61$ & 2,37 \\
& & & \\
Media s/ cultivares & 15,8 & $-2,24$ & 2,56 \\
DMS (0.05) & 1,94 & 2,25 & 0,63 \\
P (cultivar) & $* * *$ & $* * *$ & $* * *$ \\
& & & \\
Tlaltizapán & 17,2 & $-7,08$ & 3,95 \\
Poza Rica & 13,2 & 1,55 & 1,93 \\
Media s/ localidades & 15,2 & $-2,76$ & 2,94 \\
P (Localidad) & $* * *$ & $* * *$ & $* * *$ \\
P (cultivar x localidad) & + & $* * *$ & $* * *$ \\
\hline & & & \\
\hline
\end{tabular}

,$+ * * *$ se refieren a $\mathrm{P}<0.10$ y $\mathrm{P}<0.001$, respectivamente

para los coeficientes de regresión b y c (Cuadro 2) puede explicarse con las diferencias entre los patrones de humedad relativa en las dos localidades, lo que afectó los cultivares de diferente periodo de madurez de distintas maneras. Fue muy afortunado que este ensayo se realizara en dos localidades para que esos ambientes, típicos de las localidades más húmedas (PR) y más secas (TL) de Centroamérica, quedarán debidamente representados.

Conocer el contenido total de materia seca (TDM\%) también es necesario si un investigador necesita calcular la biomasa total durante la segunda mitad del llenado, cuando el contenido de materia seca del grano es significativamente mayor que el del resto de la planta (Daynard y Hunter, 1975). El cuidadoso examen de la relación humedad/peso seco de toda la planta y del grano, presentado por Daynard y Hunter (1975) y del peso seco contra R (calculado con las curvas de crecimiento presentadas por Edmeades 1972), dio como resultado una tabla de valores porcentuales de la materia 
seca para sumarlos a los valores esperados de MS\% para obtener el TDM\% (Cuadro 3). Es así que se calcula que MS\% a R=2 sea del 48\%. Para obtener el TDM\% en esta etapa, se suma el valor correspondiente del Cuadro 3 (6\%) para que resulte un TDM\% de $54 \%$.

\section{CONCLUSIONES}

El DM\% del rastrojo de maíz por encima del suelo permanece en el rango del 15-20\% hasta que esté terminada la tercera parte del llenado. Estos valores son muy constantes en todos los genotipos y localidades. Además pueden utilizarse, con toda seguridad, para convertir los pesos frescos a pesos secos en estudios en finca.

La relación entre el peso fresco y el peso seco del rastrojo varía más según el cultivar hacia el final del llenado. Esto sugiere que la mejor predicción del MS\% se obtiene por medio de las regresiones adaptadas a grupos de cultivares por separado. Para los genotipos de maduración precoz, sugerimos una ecuación de la forma $\mathrm{MS} \%$ $=12,6+0,94(\mathrm{R}) 2+1,68(\mathrm{R}) 4$ y para los genotipos de maduración tardía una ecuación de la forma $\mathrm{MS} \%=16,1$ 4,00* (R) $2+3,36(\mathrm{R}) 4$ (Figura 1). Se debe de prestar atención cuando el muestreo se lleve a cabo después de $\mathrm{R}=2,0$, ya que existe un grado de error relativamente alto relacionado con DM\% en esta etapa. La probabilidad de error es igualmente alta si las plantas están muy afectadas por la sequía, especialmente si ésta se da hacia el final del ciclo de cultivo.

\section{Protocolo sugerido}

Seleccionar aleatoriamente varias (6-8) superficies representativas en el campo para muestreo.

Identificar, marcar y medir el largo y ancho de la superficie de una parcela útil (utilizando la mitad del surco y la mitad de los espacios entre las plantas dentro de los surcos para determinar las dimensiones de la

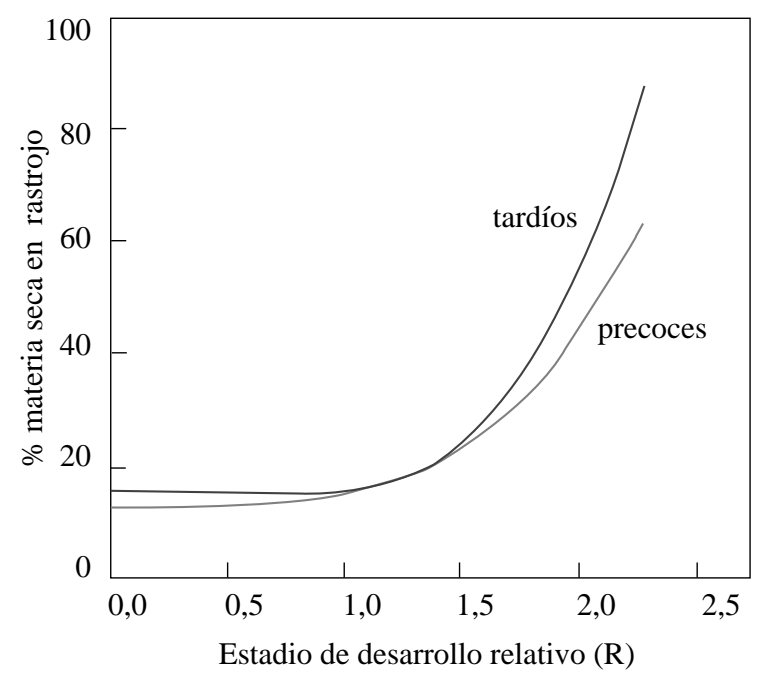

Fig. 1. Relación entre el porcentaje de materia seca en el rastrojo (\%MS) y el estadio relativo de desarrollo fenológico para cultivares precoces y tardíos de maíz.

Cuadro 3. Valores predichos del porcentaje de materia seca (\%MS) en función del estadio relativo de desarrollo (R), según coeficientes de regresión del Cuadro 2.

\begin{tabular}{lrrrrrr}
\hline Estadio relativo (R) & $\mathbf{0 . 5}$ & $\mathbf{1 . 0}$ & $\mathbf{1 . 3}$ & $\mathbf{1 . 7}$ & $\mathbf{2 . 0}$ & $\mathbf{2 . 3}$ \\
\hline dds equivalentes (PR, tardío) & 30 & 60 & 80 & 100 & 120 & 140 \\
1. Sel. Precoz C6 & 13,5 & 15,0 & 19,2 & 28,4 & 45,6 & 74,3 \\
2. Sel. Precoz C16 & 12,4 & 15,4 & 19,9 & 27,9 & 40,7 & 60,5 \\
3. La Posta C3 & 15,2 & 15,2 & 20,0 & 33,3 & 60,5 & $>100,0$ \\
4. TS6 C1 (PITS) & 15,4 & 14,6 & 18,7 & 31,5 & 58,4 & $>100,0$ \\
5. CML20 x CML27 & 14,5 & 14,4 & 18,8 & 31,5 & 57,7 & $>100,0$ \\
6. CML27 x CML52 & 15,7 & 16,1 & 20,1 & 30,3 & 50,8 & 86,2 \\
7. CML8 x CML9 & 15,2 & 15,6 & 19,3 & 29,0 & 48,2 & 81,6 \\
8. CML8 x CML22 & 15,6 & 16,6 & 20,5 & 29,7 & 47,3 & 77,3 \\
Media s/ cultivares & 15,4 & 16,1 & 19,9 & 29,3 & 47,8 & 79,5 \\
Tlaltizapán & 15,7 & 14,1 & 17,1 & 28,0 & 52,1 & 95,8 \\
Poza Rica & 13,7 & 16,7 & 22,0 & 32,4 & 50,3 & 78,8 \\
Media s/ localidades & 14,7 & 15,4 & 19,6 & 30,2 & 51,2 & 87,3 \\
\hline
\end{tabular}


Cuadro 4. Valores que añadir a los valores predichos de $\%$ MS cuando se incluye la mazorca en el estimado.

\begin{tabular}{cc}
\hline $\begin{array}{c}\text { Estadio relativo de } \\
\text { desarrollo fenológico }\end{array}$ & $\begin{array}{c}\text { Valor que añadir } \\
\text { a \% MS }\end{array}$ \\
\hline 1,2 & 0 \\
1,3 & 1 \\
1,4 & 2 \\
1,5 & 3 \\
1,6 & 4 \\
1,7 & 5 \\
1,8 & 6 \\
1,9 & 7 \\
2,0 & 6 \\
2,1 & 6 \\
2,2 & 6 \\
2,3 & 5 \\
2,4 & 5 \\
2,5 & 4 \\
10 & 8 \\
\hline
\end{tabular}

parcela recolectada). Registrar la superficie de la parcela recolectada (PA) en unidades de $\mathrm{m}^{2}$.

Cortar las plantas dentro de la superficie de muestra marcada por encima del suelo y juntarlas en un hato. Anote la hora.

Cuando hayan pasado 10 minutos después del corte, tomar el peso fresco en $\mathrm{kg}$ con una báscula de campo. Registrar el peso y la fecha.

Registrar los días transcurridos desde la siembra hasta la antesis (AD) del cultivar y desde la siembra hasta la recolecta de muestra (SHD); calcular R como (AD/ SHD).
Calcular MS\% con la ecuación apropiada referida en la "Conclusión 2". Si se necesita el MST\%, sumar al valor calculado de MS\% a la corrección apropiada para la etapa de crecimiento en la Cuadro 4.

Convertir el peso fresco (PF) a peso seco de rastrojo (PS; $\mathrm{kg}$ ) de la siguiente manera: $\mathrm{PS}=\mathrm{PF} * \mathrm{MS} \%$. Para obtener el peso seco total, incluyendo las mazorcas, convertir el peso fresco total (PFT) a peso seco total (PST; $\mathrm{kg}$ ) de la siguiente manera: PST=PFT*MST\%. Calcular materia seca por unidad de área (ton ha-1) de la siguiente manera, PS*10/PA.

Promediar la producción de peso seco de cada una de las seis a ocho sub muestras para obtener una muestra representativa de todo el campo.

\section{LITERATURA CITADA}

BOLAÑOS, J. 1995. Physiological bases for yield differences in selected maize cultivars from Central América. Field Crops Res. 42: 69-80.

DAYNARD, T.B.; HUNTER, R.B. 1975. Relationships among whole-plant moisture, grain moisture, dry matter yield, and quality of whole-plant corn silage. Can. J. Plant Sci. 55:77-84.

EDMEADES, G.O. 1972. Maize in the Manawatu: A field study of the effects of spacing and variety upon the growth of Zea mays L. M.Agric. Sci. Tesis, Massey University, New Zealand. 123pp.

FAIREY, N.A. 1980. The effects of hybrid maturity, date of planting, and date of harvesting on growth and development of forrage maize. Can. J. Plant Sci. 60:1367-1375.

LOOMIS, R.S.; CONNOR, D.J. 1992. Crop Ecology: Productivity and Management of Agricultural Systems. Cambridge University Pres, UK. 538 pp.

LUDLOW, M.M.; MUCHOW, R.C., 1990. A critical evaluation of traits for improving crop yields in water-limited environments. Adv. Agron. 43:107-153. 